\title{
Broadband Active Differential Array for the Mid-Frequency SKA Band
}

\author{
L. E. García-Muñoz ${ }^{1}$, D. Segovia-Vargas' ${ }^{1}$, O. Garcia-Perez', E. Ugarte-Muñoz ${ }^{1}$, \\ V. González-Posadas' ${ }^{2}$, J. M. Serna-Puente ${ }^{3}$, and J. A. Lopez-Fernandez ${ }^{3}$ \\ ${ }^{1}$ Department of Signal Theory and Communications \\ Carlos III University in Madrid \\ Avenida de la Universidad 30, 29811, Leganés, Madrid, Spain \\ E-mail: legarcia@tsc.uc3m.es; dani@tsc.uc3m.es; ogarpe@tsc.uc3m.es; eugarte@tsc.uc3m.es \\ ${ }^{2}$ Department of Ingeniería Audiovisual y Comunicaciones \\ Polytechnic University of Madrid (UPM) \\ 28031, Madrid, Spain \\ E-mail:vgonzalz@diac.upm.es \\ ${ }^{3}$ Instituto Geográfico Nacional in Yebes \\ Observatorio Astronómico Nacional \\ E-mail: jm.serna@oan.es; ja.lopez@oan.es
}

\begin{abstract}
This paper presents the design and characterization process of an active array demonstrator for the mid-frequency range (i.e., $300 \mathrm{MHz}-1000 \mathrm{MHz}$ ) of the future Square Kilometre Array (SKA) radio telescope. This demonstrator, called FIDA3 (FG-IGN: Fundación General Instituto Geográfico Nacional - Differential Active Antenna Array), is part of the Spanish contribution for the SKA project. The main advantages provided by this design include the use of a dielectricfree structure, and the use of a fully-differential receiver in which differential low-noise amplifiers (LNAs) are directly connected to the balanced tapered-slot antennas (TSAs). First, the radiating structure and the differential low-noise amplifiers were separately designed and measured, obtaining good results (antenna elements with low voltage standingwave ratios, array scanning capabilities up to $45^{\circ}$, and noise temperatures better than $52 \mathrm{~K}$ with low-noise amplifiers at room temperature). The potential problems due to the differential nature of the proposed solution are discussed, so some effective methods to overcome such limitations are proposed. Second, the complete active antenna array receiving system was assembled, and a $1 \mathrm{~m}^{2}$ active antenna array tile was characterized.
\end{abstract}

\section{Introduction}

$\mathbf{T}$ he Square Kilometre Array (SKA) will be a next-generation radio telescope with a collecting area of about $1 \mathrm{~km}^{2}$ $[1,2]$. This instrument is intended to cover the frequency range between $70 \mathrm{MHz}$ and $10 \mathrm{GHz}$, and it will be much more sensitive than current telescopes. The SKA technology will allow breakthroughs in different areas of science, such as astro-particle physics and cosmology, fundamental physics, galactic and extragalactic astronomy, solar-system science, and astrobiology. In the context of radio-astronomy instrumentation, differ- ent antenna technologies are proposed in order to cover the entire bandwidth: arrays of dipole-like antennas in random sparse configurations for the lowest frequency band ( $70 \mathrm{MHz}$ to $450 \mathrm{MHz}$ ), aperture arrays of tapered-slot antennas (TSAs) for the mid-frequency range ( $300 \mathrm{MHz}$ to $1000 \mathrm{MHz}$ ), and small dish reflectors at higher frequencies ( $1 \mathrm{GHz}$ to $10 \mathrm{GHz}$ ).

There is a global collaboration among more than 20 countries that are currently working to find the most-suitable technologies needed to construct this enormous instrument. The SKA will be formed by a central core and by several sub- 
stations along five spiral arms out to $200 \mathrm{~km}$ radius. The construction is scheduled to begin in 2016 , and initial observations are projected to begin in 2019. The instrument is intended to be fully operational by 2024 . Nowadays, the research groups and institutions involved are investigating the technologies that will be necessary to fulfill both the technical and cost requirements.

In the case of the mid-frequency arrays, $66 \%$ of the antennas will be placed in the central core, and the rest will be placed along the spiral arms. Above $300 \mathrm{MHz}$, the sky noise is relatively low and flat, so the system noise temperature is mainly determined by the array's technical performance. A dense configuration (i.e., where the distance between elements is shorter than a half-wavelength) thus seems the most adequate choice for the highest dynamic range [3]. Additionally, a dense configuration provides fixed effective aperture across the band, wider beams, faster survey speeds, easier beamforming, and absence of grating lobes. Each mid-frequency aperture subarray will consist of around 75,000 dual-polarization elements, and will have a diameter of $180 \mathrm{~m}$. The entire telescope will consist of around 250 mid-frequency subarrays.

During recent years, several dense-antenna-array demonstrators have been proposed for the mid-frequency band of the SKA [4-8]. The intrinsic nature of all the proposed antenna elements is balanced (i.e., the signal is defined as the signal between two identical conductors). First prototypes were based on Vivaldi-type antennas, with a balancing feeding board (balun) to convert the balanced signal coming from the antenna into a ground-referred (single-ended) signal [4-6]. This therefore allowed using conventional single-ended amplifiers to amplify the weak signals captured by each antenna element. This approach has been seen to be inefficient, since the losses of the passive balun directly contribute to increasing the overall system noise temperature. Novel approaches considered a fully-differential front-end, based on a differential low-noise amplifier (LNA), directly connected to the antenna [7, 8]. Another important aspect is the cost, which is directly related to the materials and the fabrication process of the antennas. First designs were based on printed antennas on FR4-substrate boards, due to the ease of fabrication [4]. Novel designs avoid the use of substrate boards for printing the antennas, due to their high cost and potential losses. In contrast, they are directly manufactured in aluminum [5-7], or make use of cheap foils or foam structures [8]. The work presented in this paper is focused on the design and characterization of a broadband dielectric-free differential active-antenna-array demonstrator, the technology of which is proposed as a potential candidate for the mid-frequency range $(300 \mathrm{MHz}$ to $1000 \mathrm{MHz}$ ) of the SKA telescope.

\section{Radiating Structure}

\subsection{Antenna Array Design}

From the wide variety of tapered-slot antenna elements, the so-called bunny-ear type was the one used in the design of this array prototype, since the two thin parallel feeding lines allowed a direct integration with the differential low-noise amplifiers. A representation of the bunny-ear antenna element and the proposed dual-polarized-array configuration are shown in Figure 1. In this case, the antenna element was completely built in aluminum, avoiding the use of a potentially lossy dielectric. Furthermore, the antenna was fed with a balanced signal between both antenna ports. This antenna array was a dense array, and was characterized in terms of the active reflection coefficient, which takes into account the mutual coupling effects between the antenna elements, and allows obtaining an equivalent model of the system with uncoupled channels $[9,10]$. In dense arrays, it is essential to deal with the mutual coupling, since the constructive contribution of the mutual coupling helps to achieve a suitable VSWR and facilitates the broadband performance of the array, the beamforming, and the absence of grating lobes. However, the destructive

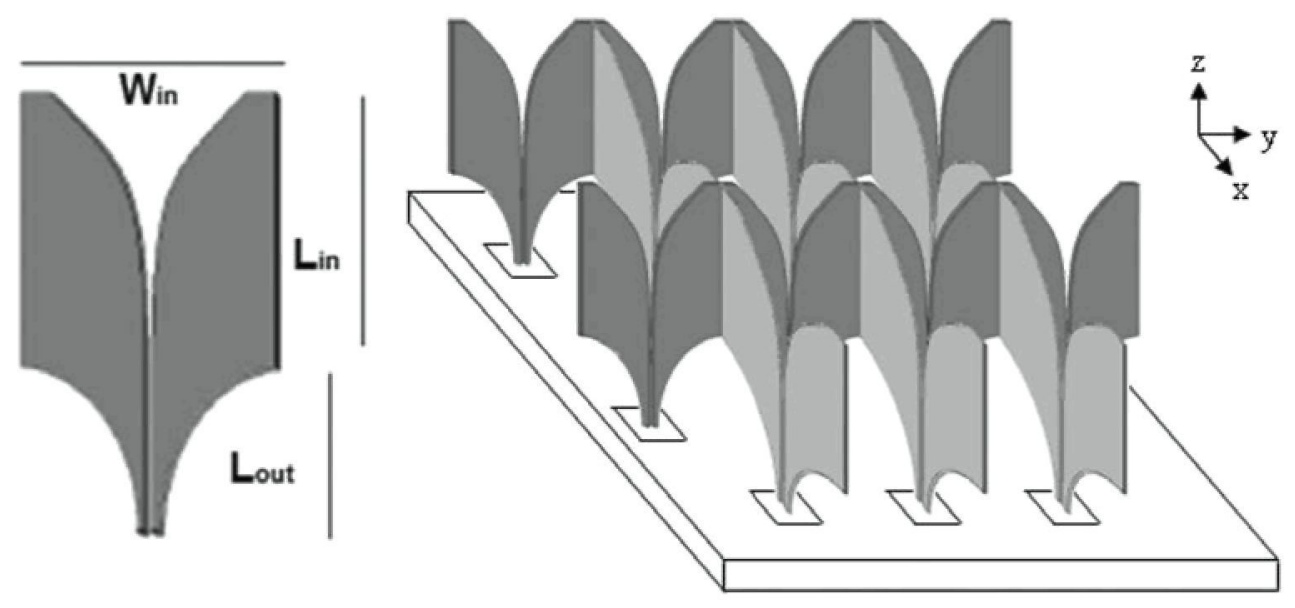

Figure 1. The bunny-ear antenna element and array configuration. 
Table 1. The dimensions of the optimum bunny-ear antennas in the array.

\begin{tabular}{|c|c|c|c|}
\hline $\begin{array}{c}\text { Width, } \\
W_{\text {in }}\end{array}$ & $L_{\text {in }}$ & $L_{\text {out }}$ & Spacing \\
\hline \hline $168 \mathrm{~mm}$ & $350 \mathrm{~mm}$ & $77 \mathrm{~mm}$ & 0 \\
\hline
\end{tabular}

contribution implies an increase in the VSWR that makes scan blindness rise for some particular frequency and some particular angle.

The active reflection coefficient should be acceptably well matched (in this case, the reference impedance was chosen as $150 \Omega$ in differential mode) over the entire frequency band of interest and for an array-scanning range from broadside up to $45^{\circ}$, in order to obtain a homogeneous system noise temperature when connecting the differential low-noise amplifiers to each element of the antenna structure. For the subsequent analysis and optimization process, the array was assumed to be infinite, so that the active reflection coefficients for all the elements were identical, and the array could be characterized by a unique reflection-parameter curve for a certain array-scanning condition. Regarding the large dimensions of the mid-frequency SKA stations, this assumption seemed reasonable.

The aperture efficiency is given as

$$
A_{\text {eff }}=a b \eta_{\text {rad }} \cos \theta \text {, }
$$

where $a b$ is the dimension of the unit cell, $\theta$ is the incidence angle, and $\eta_{\text {rad }}$ is the unit-cell radiation efficiency. For the values in Table 1, the unit-cell dimensions were $28.6 \times 10^{-3} \mathrm{~m}^{2}$. Table 1 gives the data for the dimensions of the array of bunnyear antennas. $W_{\text {in }}$ is the width, and $L_{\text {in }}$ and $L_{\text {out }}$ are the dimensions of the upper and bottom vertical profiles and the spacing between the antennas.

A parametric study of the main antenna parameters was carried out, in order to obtain a VSWR $<2.5$ over the band of interest [11]. During the optimization process, some impedance anomalies were observed in the scan impedance response when the array was scanning at off-broadside angles, as shown in Figure 2. This effect was attributed to the differential nature of the bunny-ear antennas in the presence of a third conductor (the ground plane) [11]. There exist some combinations of frequency and scan-angle values at which the common-mode currents dominated the differential-mode currents, leading to a surge in the radiated power, and therefore causing a strong mismatch in the differential port and the corresponding scan-blindness effect.

Figure 3 illustrates this effect: a plane wave incident on the antenna array was simulated to show the induced surface currents on the antenna's surface. At the frequencies without the presence of anomalies, odd-mode currents were observed in the antennas, as shown in Figure 3a. However, at the frequencies and scan angles at which the anomaly was predicted from the scan impedance curve, the structure propagated dominating even-mode currents, as shown in Figure $3 b$.

In order to mitigate the effect of the even-mode currents, a solution based on connecting two resistors between each one of the feeding lines of the antennas and the ground plane was proposed (see Figure 3c). In this way, the common-mode current suffered a stronger suppression than the differential-mode current, since the two resistors were seen as being in series for the differential-mode excitation, whereas they were in parallel for the common-mode excitation (a factor of four difference) Since the resistors are noisy elements, the noise contribution as a function of the resistor value was studied (see Figure $3 \mathrm{~d}$ ). The best trade-off was reached when the resonance in the differential-scan impedance became sufficiently suppressed while the overall impedance characteristics remained almost unaf-

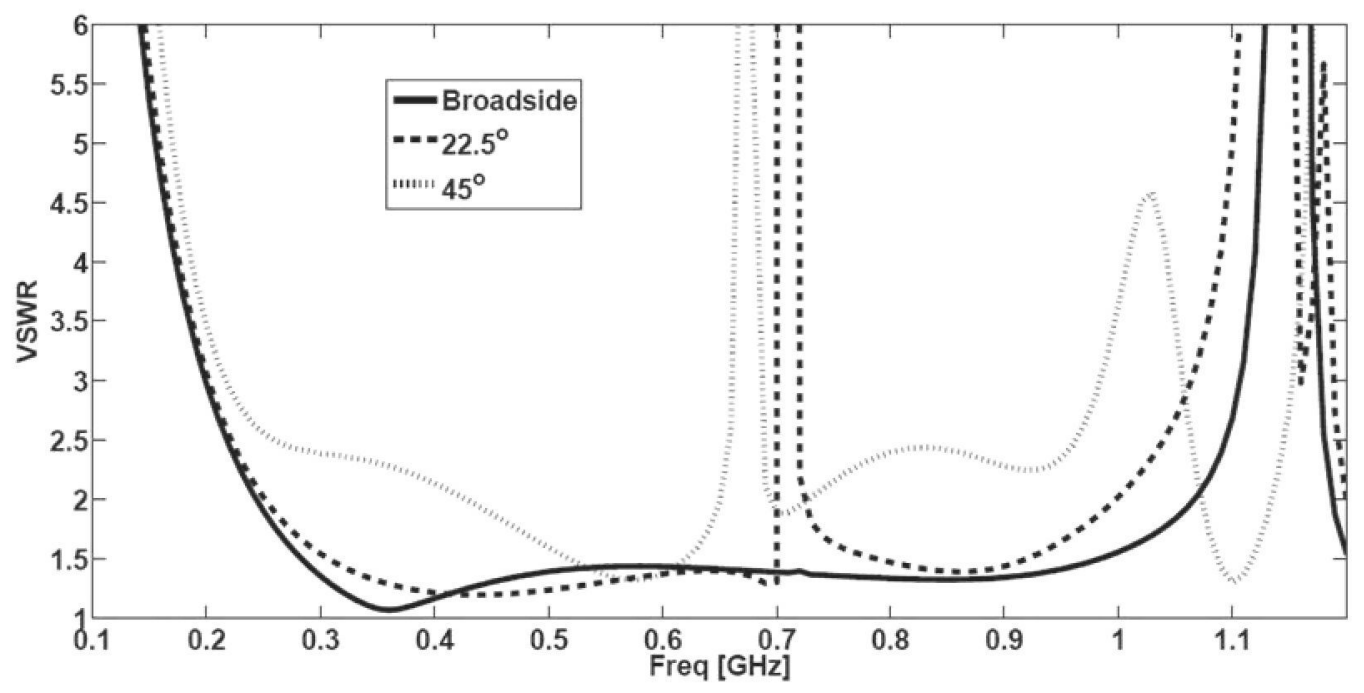

Figure 2. The simulated E-plane VSWR curves, where anomalies appeared when scanning at off-broadside angles. 

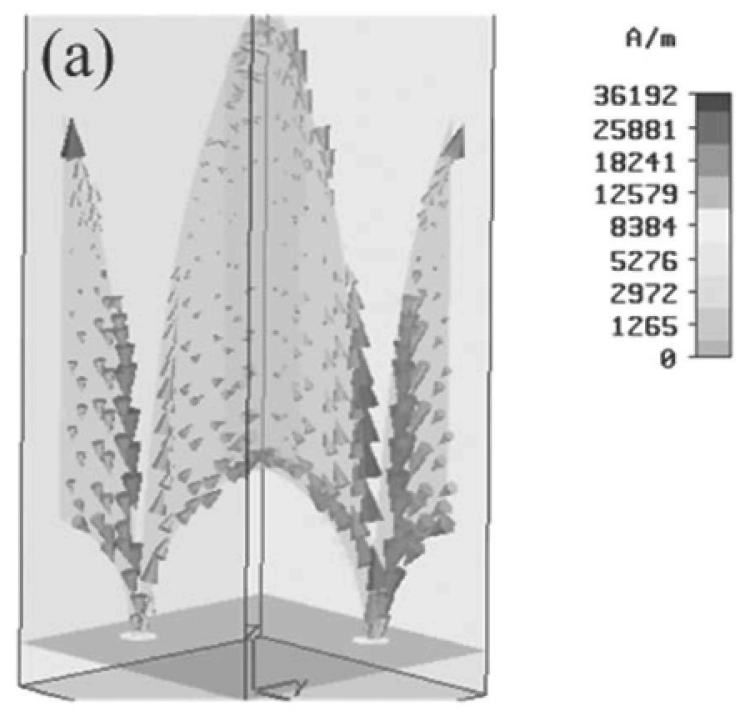

Figure 3a. Odd-mode currents in the antenna at $500 \mathrm{MHz}$.
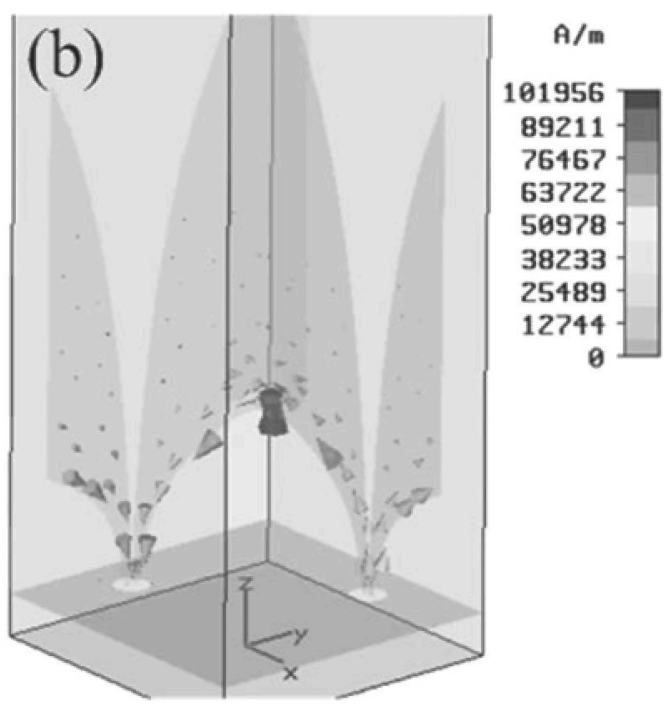

Figure $3 b$. Even-mode currents at the frequency of the anomaly.

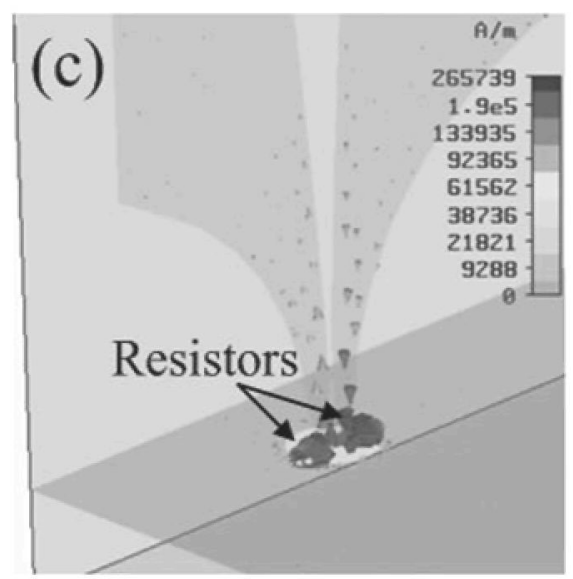

Figure 3c. The proposed solution, based on resistive mitigation.

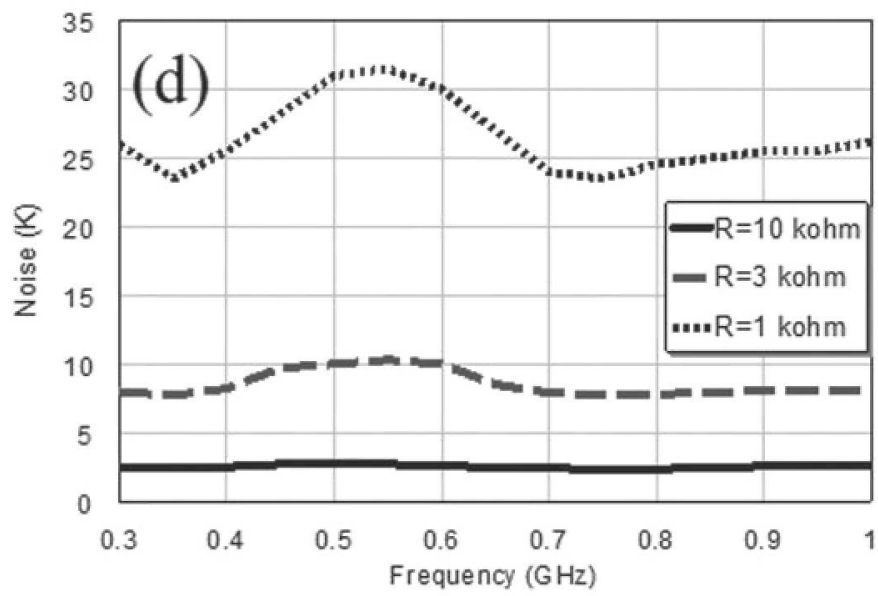

Figure 3d. The noise contribution of the padding resistors.

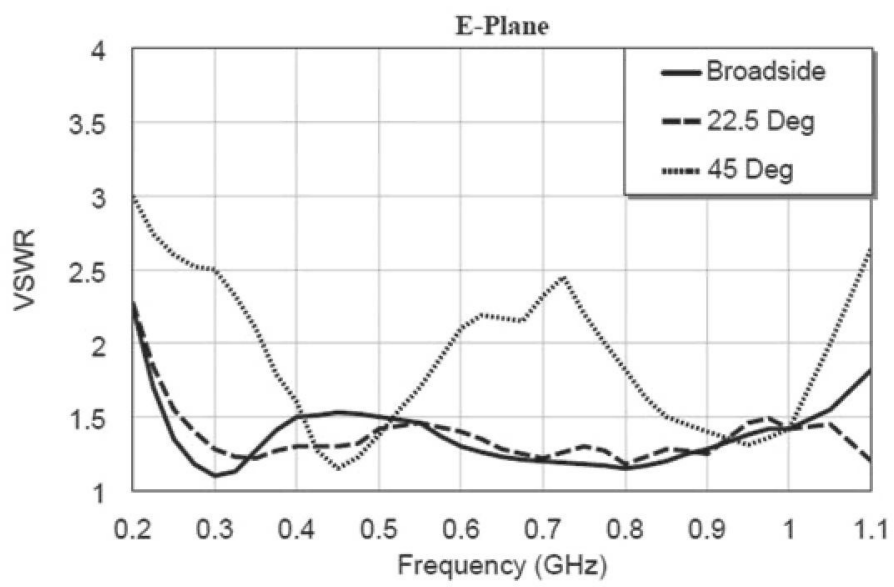

(a)

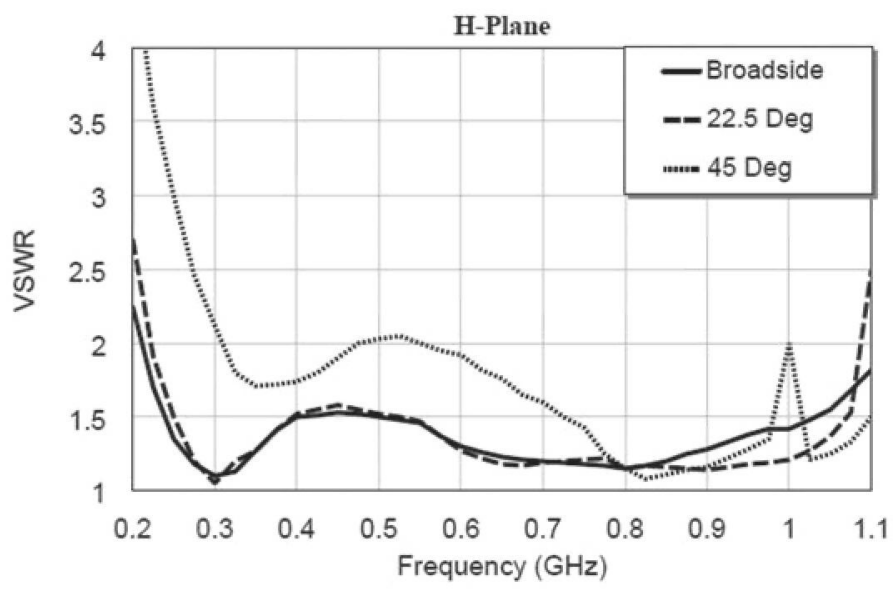

Figure 4. The simulated VSWR curves when using a resistor value of $3 \mathrm{k} \Omega$ to correct the anomalies for both (a) E-plane and (b) H-plane scanning. 
fected. A $3 \mathrm{k} \Omega$ resistance was chosen as an appropriate tradeoff value for the final prototype, which implied an extra contribution of around $10 \mathrm{~K}$ to the total noise temperature of the system. The final simulated VSWR curves of the infinite array for both E- and H-plane scanning are shown in Figure 4, in which the condition VSWR $<2.5$ was fulfilled in the band of interest.

The antenna's impedance matching degraded by increasing the resistor values beyond a certain point as the effect of the lumped resistors decreased [11], i.e., on transmitting, a lower power was delivered to these resistors because of a lowpower transmission factor from the differential antenna port to the resistors. Likewise, on receiving, only a small part of the thermal noise that was generated by these resistors will be observed at the antenna port, due to this low transmission factor. Finally, the antenna gain was not greatly affected, since the padding resistors were simultaneously included in the differential broadband amplifier through RF chokes, preventing its effect at RF frequencies.

Finally, a guideline for the design of the active receiving array is given as follows:

- Design the radiating element (the bunny-ear antenna) inside the dense array with the optimum dimensions to reduce the scan blindness and optimize the aperture efficiency [11]

- Calculate and measure the active reflection coefficient.

- Design the low-noise differential amplifier (DLNA) for the impedance corresponding to the active reflection coefficient.

- Integrate the antenna with the low-noise differential amplifier and measure the active receiving antenna.

\subsection{Antenna Array Measurement}

An array prototype formed by 64 antenna elements was manufactured and characterized (see Figure 5). The complete scattering $(S)$ parameter matrix of the array was measured, in order to obtain all the mutual-coupling coefficients between all the antenna elements. Since the antennas were fed in differential mode, passive baluns connected to the bunny-ear antennas were used to convert from differential to single-ended mode, and to directly use a two-port network analyzer. By mathematically processing this $S$-parameter matrix, it was possible to calculate the active reflection coefficient of any element in the array [9]:

$$
\Gamma_{a c t}^{i}=\sum_{j=1}^{N} s_{j i}^{a n t} e^{j\left(\phi_{j}-\phi_{i}\right)},
$$

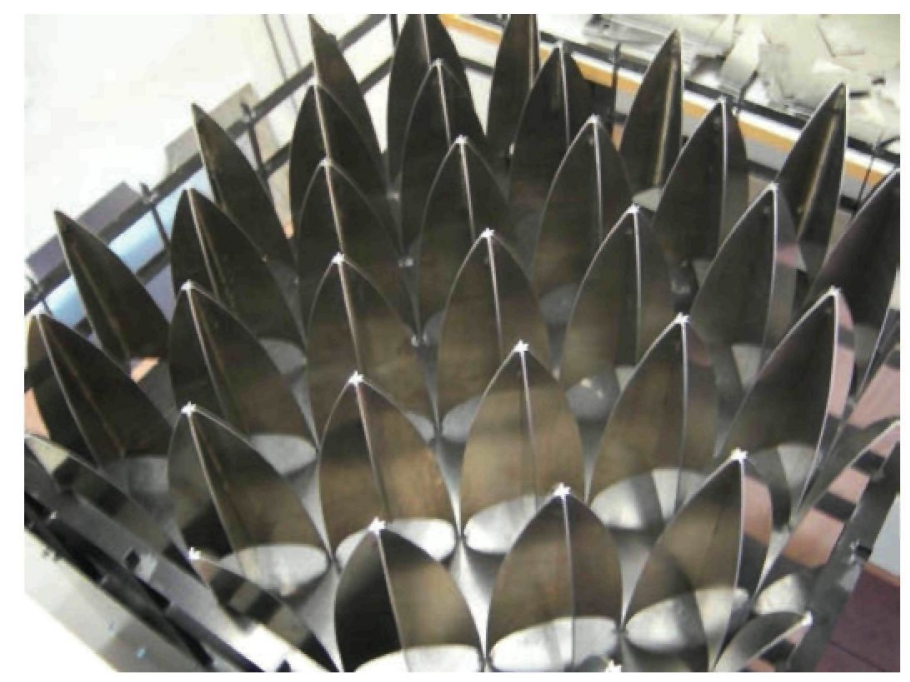

Figure 5. A photograph of the manufactured antenna-array prototype.

where $\Gamma_{a c t}^{i}$ is the active reflection coefficient of the $i$ th element, $N$ is the number of elements in the array, the $s^{\text {ant }}$ are the $S$ parameters of the radiating structure, and $\phi_{i}$ is the phase delay in the $i$ th path (used for scanning). The measured active reflection coefficient of the center element of the manufactured structure, assuming different scan angles from broadside up to $45^{\circ}$ in both the $\mathrm{E}$ and $\mathrm{H}$ planes, is shown in Figure 6. The feeding network was designed by making use of commercial broadband power combiners (JPCS-8-10 and JS4PS-1W from Mini-Circuits) and commercial broadband phase shifters. First, four $8: 1$ power combiners combined the 32 equal polarized antennas into four branches. Second, one 4:1 power combiner combined into a single output port.

In the case of broadside scanning, the measured VSWR remained below the desired value of 2.5 in the band of interest. For scanning angles close to the limit of $45^{\circ}$, the VSWR performance degraded in the upper part of the band. These results were acceptable, taking into account the small dimensions of the implemented prototype. Better results could be expected with a larger implementation of the array.

\section{Differential Amplifiers}

\subsection{Low-Noise Amplifier Design}

As was explained before, bunny-ear antennas provide a balanced output. Two options may thus be considered in a receiver configuration. The first option is using a passive balun to convert the incoming balanced signal into a signal in singleended mode (one-port output), and to then connect a conventional low-noise amplifier. However, this option may be strongly limited by the losses and bandwidth restrictions of the passive 
balun. In this first approach, the losses of the balun directly contribute as a noise increase in the system's performance. The second option, which is the one proposed in this work, consists of using a differential low-noise amplifier directly connected to the antenna, working as an active balun.

The circuit schematic of the proposed differential lownoise amplifier is shown in Figure 7 [12]. It consisted of two cascaded differential-pair stages. This configuration provided protection against external interference due to the mitigation of common-mode signals [13]. The sources of the transistors in both branches were connected to a common current source, which was implemented by using a transistor in series with an inductance. Some padding resistors were included in the design in order to ensure the unconditional stability of the amplifier.

\subsection{Low-Noise Amplifier Measurements}

The differential low-noise amplifier prototype is shown in Figure 8 . It was implemented by using discrete surface-mount components on a microstrip substrate. The transistors were lownoise PHEMTs, model ATF-34143 from Avago Technologies. A passive transformer was connected at the output of the circuit in order to provide a single-ended output port.

The characterization at microwave frequencies of this type of differential devices required some specific methods, and it was especially critical for the case of the noise. In this case, the source impedance seen by the low-noise amplifiers was assumed to be $150 \Omega$ in differential mode (average scan impedance of the antennas). The gain was thus obtained by means of the so-called mixed-mode scattering $(S)$ parameters

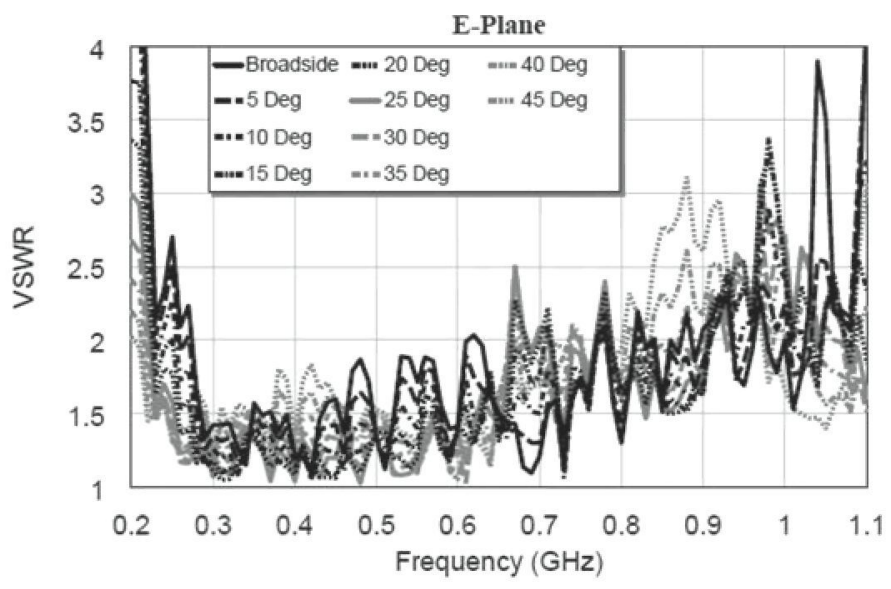

(a)

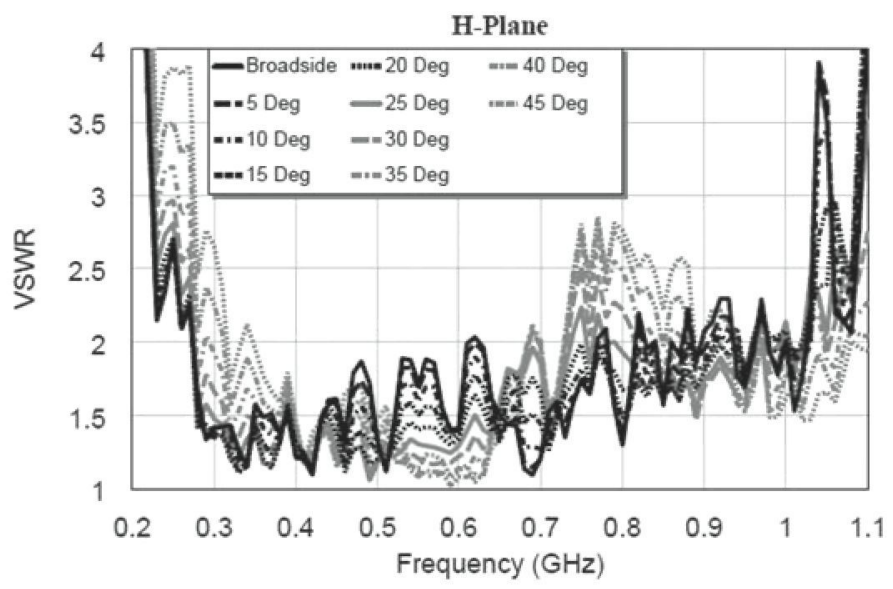

(b)

Figure 6. The measured VSWR curves of the center element of the manufactured 64-element array for different (a) E-plane and (b) H-plane scanning.

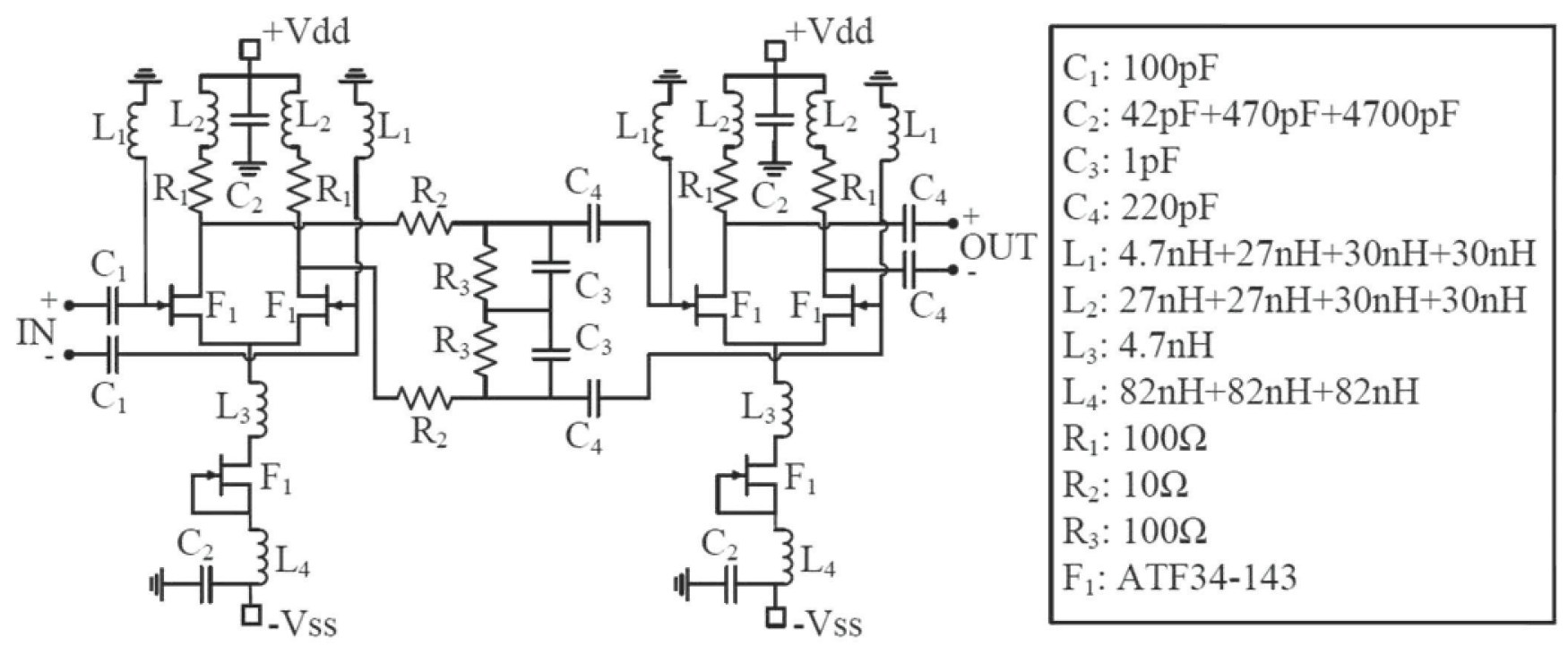

Figure 7. The electrical schematic of the proposed differential low-noise amplifier. 


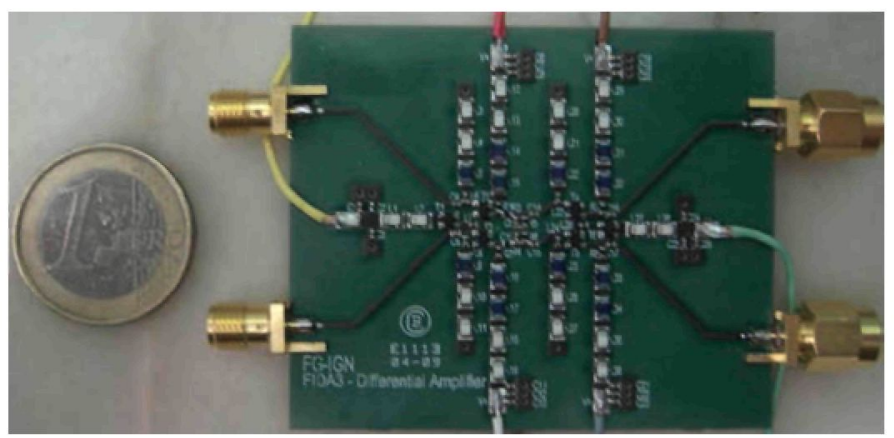

Figure 8. The differential low-noise-amplifier prototype board.

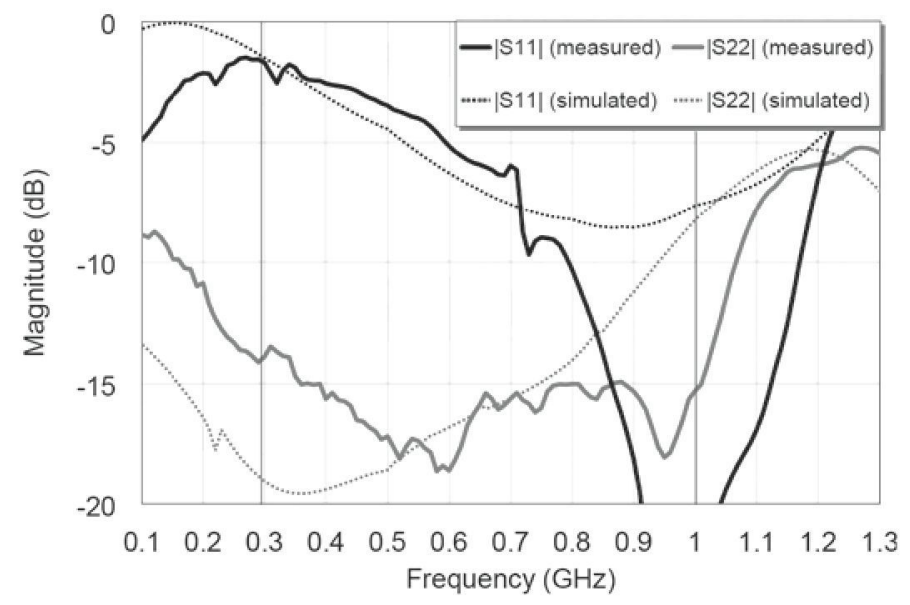

Figure 9. The measured and simulated input and output return losses of the differential low-noise amplifier.

[13]. The mixed-mode $S$ parameters allow obtaining an equivalent representation of the circuit as a two-port device, considering the input ports to be a single port that can be excited with differential or common-mode signals. The input and output return losses of the three-port differential low-noise amplifier can be calculated from the standard $S$ parameters as [13]

$$
s_{d d 11}^{m m}=\frac{1}{2}\left(s_{11}^{s t d}-s_{12}^{s t d}-s_{21}^{s t d}+s_{22}^{s t d}\right),
$$

$s_{s s 22}^{m m}=s_{33}^{s t d}$,

where the single-ended input ports are numbered as 1 and 2 (assuming a $75 \Omega$ port impedance for each port), the output port is numbered 3 ( $50 \Omega$ port impedance), $s^{\text {std }}$ are the standard $S$ parameters, $s^{m m}$ are the mixed-mode $S$ parameters, subscript $d$ denotes differential mode, and subscript $s$ denotes single-ended mode. These two parameters are shown in Figure 9. It could be seen that the return losses at the input were quite poor in the lower part of the band. This was due to the high input impedance provided by the transistors at some hundreds of MHz. In this case, an input-matching network was not used, in order to avoid the potential losses of a passive circuit at the input of the amplifier. In the case of the output return losses, they were below $-13 \mathrm{~dB}$ in the band of interest. The differential gain can also be obtained from the corresponding mixed-mode $S$ parameter as

$$
s_{d s 21}^{m m}=\frac{1}{\sqrt{2}}\left(s_{31}^{s t d}-s_{32}^{s t d}\right)
$$

where the notation is the same as in Equation (2). As could be seen from Figure 10, the gain was higher than $26 \mathrm{~dB}$ in the band of interest.

In the case of the noise figure, the hot/cold method using a differential load, presented in [14], was applied. It required connecting a resistor with the value of the desired differential source impedance ( $150 \Omega$, in this case) at the input of the differential amplifier. This connection was done by means of a ladder line with the same characteristic impedance as the load. With this configuration, the output power of the amplifier was obtained for two different conditions: when the load was at ambient temperature (i.e., hot measurement: $T_{\text {hot }} \approx 290 \mathrm{~K}$ ), and when the load was dipped into liquid nitrogen (cold measurement: $T_{\text {cold }} \approx 70 \mathrm{~K}$ ), obtaining the corresponding hot and cold output powers ( $P_{\text {hot }}$ and $P_{\text {cold }}$ ). The noise temperature of the device under test (DUT) can be calculated by the Y-factor method as [15]

$$
T_{D U T}=\frac{T_{h o t}-Y T_{\text {cold }}}{Y-1}
$$

where $Y=P_{\text {hot }} / P_{\text {cold }}$. The noise temperature obtained from this method is shown in Figure 10. The noise was lower than $52 \mathrm{~K}$ in the band of interest.

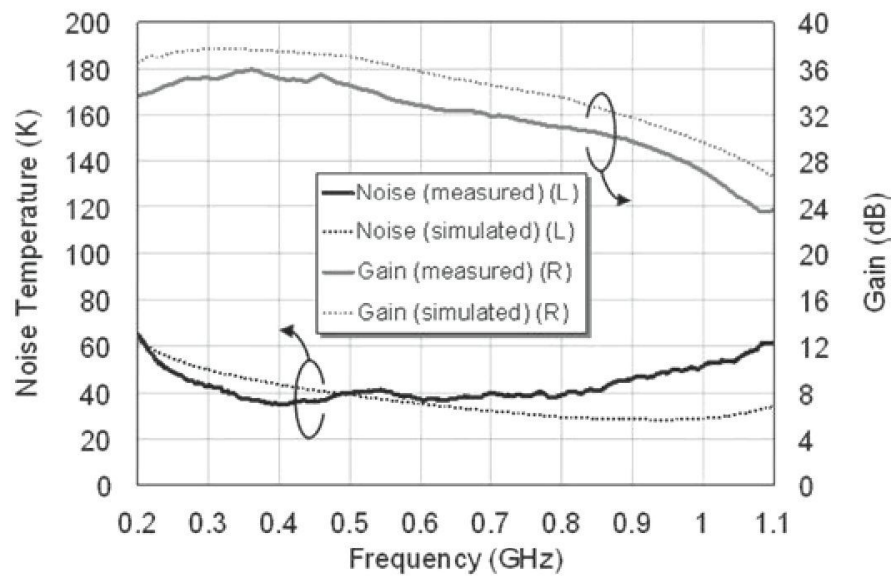

Figure 10. The measured and simulated gain and noise performance of the differential low-noise amplifier. 


\section{System Integration}

Once the separated performance of both the radiating structure and the differential low-noise amplifiers was characterized, the next step consisted in the integration of all these components, forming an active antenna-array receiver. The antennas were placed over a metallic layer, which worked as the ground plane of the array. The differential ports of the antennas passed the ground plane through holes in the plane. The differential low-noise amplifiers were then soldered to each antenna below the ground plane, as is shown in Figure 1la. Each differential low-noise amplifier provided a single-ended output (because of the passive balun at the output, as shown in Figure 8). The outputs of the 32 elements for each polarization were thus combined by using commercial broadband combiners (i.e., four $8: 1$ and one $4: 1$ devices), obtaining a single coaxial output per polarization. The connections between the amplifiers and the combiners was done by means of flexible coaxial cables.

The noise characterization of the array system was done in a hot/cold system facility developed by ASTRON (The Netherlands) [16]. This system consisted of a large metallic box with a removable roof that allowed characterizing the noise temperature of many types of receiving systems. A photograph of the FIDA3 prototype inside the hot/cold system is shown in Figure 12. The aim with the lateral metallic walls is to isolate the system under test from the undesired terrestrial interference. The characterization of the system was based on the Y-factor method [15]. In fact, the procedure was equivalent to the procedure followed in the characterization of the lownoise amplifiers, but on a larger scale. The output power of the system was measured when the system was excited through two different source temperatures. For this case, the cold test was done when the system was looking at the sky, and the hot test was obtained when the array was looking toward an absorbing material, which was placed in the removable roof. The cold temperature was given by the average sky noise temperature (which is frequency dependent), and the hot temperature was the ambient temperature. With the two temperatures and the two corresponding output powers, the system noise temperature could be calculated by using Equation (4).

In order to obtain higher levels of power with the spectrum analyzer, a broadband low-noise amplifier was connected at the output of the array. In addition, a tunable bandpass filter was connected before the output amplifier, in order to avoid out-of-band interference that could saturate such an amplifier. The measurement along the band of interest was thus undertaken by means of spectrum captures in subbands of $20 \mathrm{MHz}$, with frequency steps of $10 \mathrm{MHz}$. The measured system noise temperature of the implemented active array tile is shown in Figure 13. This system noise value was the average noise temperature of the 32 elements [17]. The gray curve shows the noise-temperature curves directly obtained from each one of the subbands in which the measurement was done. The continuous black line corresponds to the average noise-temperature value in the frequency ranges in which the absence of interference allowed a reliable acquisition of the noise-temperature level.

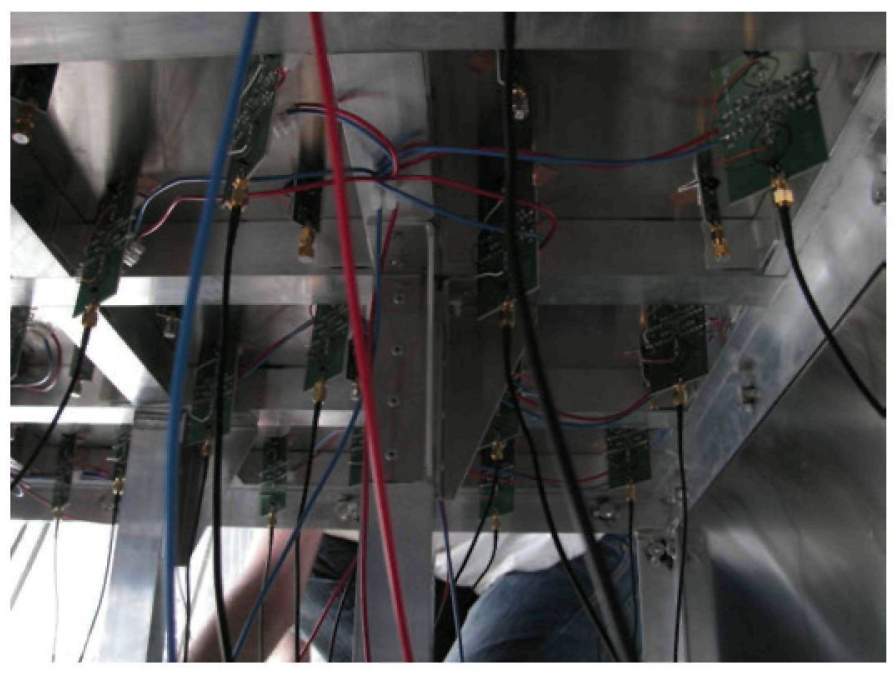

Figure 11. The differential low-noise amplifiers connected to the antennas below the ground plane in the implemented array prototype.

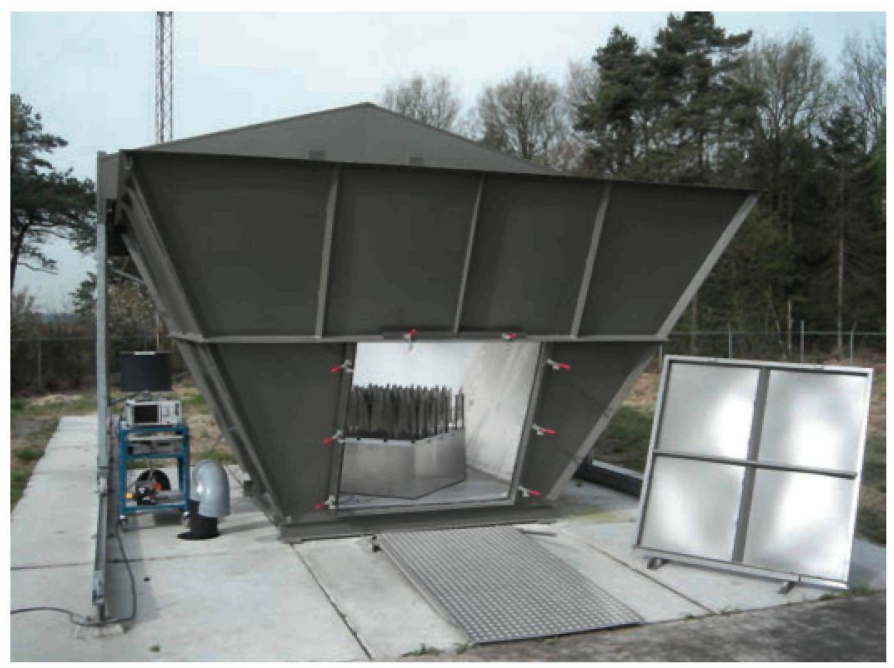

Figure 12. Measurements of the gain and noise performance of the differential low-noise amplifier.

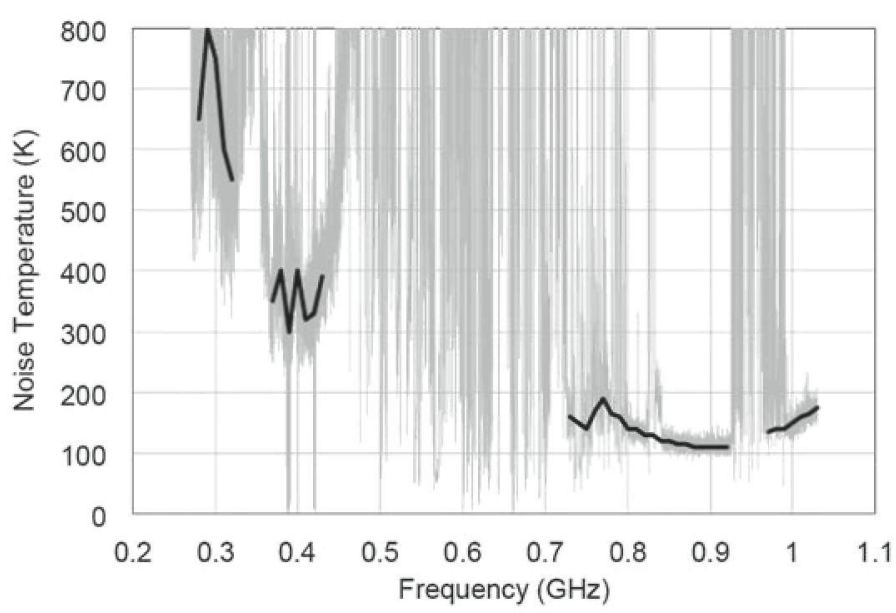

Figure 13. The measured noise temperature of the FIDA3 array tile. The gray curve shows the raw data, and the black line represents the average value in the frequency ranges with low interference. 
There were three frequency ranges, (i.e., $330 \mathrm{MHz}$ to $360 \mathrm{MHz}$, $440 \mathrm{MHz}$ to $720 \mathrm{MHz}$, and $930 \mathrm{MHz}$ to $960 \mathrm{MHz}$ ), in which the interference - presumably coming from TETRA, TV, GSM900, and other services - make a correct acquisition of the system noise temperature impossible.

Regarding the measured performance of the implemented antenna-array tile (Figure 13), the system noise temperature was around $700 \mathrm{~K}$ in the lower part of the band, and decreased down to around $100 \mathrm{~K}$ in the higher part of the band. These noise values differ significantly with respect to the $55 \mathrm{~K}$ measured from the isolated amplifier with the ideal $150 \Omega$ sourceimpedance condition (Figure 10). This disagreement can be attributed to different effects. In the higher part of the band, the system noise temperature was around $100 \mathrm{~K}$ to $150 \mathrm{~K}$, and the noise temperature of the low-noise amplifier was around $50 \mathrm{~K}$. In this range, the discrepancy could be attributed to the mismatching introduced by the edge elements, the low-noise amplifiers of which see a source impedance far from the desired $150 \Omega$. It should be noticed that 14 of the 32 elements were placed at the edge of the grid, whereas the antenna optimization was carried out assuming an infinite-by-infinite implementation. In the case of the lower part of the band, the discrepancy was much more significant, since the system noise temperature reached up to $800 \mathrm{~K}$ in the worst case. One of the reasons for the larger system noise temperature at low frequencies was that the array was electrically smaller (in terms of wavelength) and, therefore, further from the infinite-array assumption. Furthermore, the shortest electrical distance between the elements at lower frequencies makes the coupling coefficients larger in magnitude, so removing the adjacent elements (as in the case of the edge elements) usually makes results more critical. Furthermore, the noise resistance, $R_{n}$, of the transistors used in the amplifier design was higher in the lower part of the band, so the low-noise amplifiers were less robust against antenna impedance-mismatching in this range.

Beyond the previous aspects, another effect, related to the influence of the input impedance of the low-noise amplifier on the active reflection coefficient of the antennas, was identified. Regarding Equation (1), the active reflection coefficient of an antenna element is dependent on the $S$ parameters of the antenna structure. However, as it was stated in [10], this expression is only valid assuming perfect input-impedance matching in the low-noise amplifiers. The dependence of the active reflection coefficient on the input impedance of the low-noise amplifiers is thus intrinsically present, since the port impedance (for which the $S$ parameters of the radiating structure are defined) should be adjusted to equal the input impedance of the lownoise amplifiers. This dependence on the input impedance of the low-noise amplifier was explicitly given in [18], in which the active antenna impedance of any element in an $N$-element antenna-array structure is defined as

$$
Z_{a c t}^{i}=\frac{\mathbf{i}_{\bullet \mathbf{i}}{ }^{T} \cdot \mathbf{Z}^{a n t} \cdot\left(\mathbf{Z}^{a n t}+Z_{i n}^{L N A} \cdot \mathbf{I}\right)^{-1} \cdot \mathbf{w}}{\mathbf{i}_{\bullet \mathbf{i}}{ }^{T} \cdot\left(\mathbf{Z}^{a n t}+Z_{i n}^{L N A} \cdot \mathbf{I}\right)^{-1} \cdot \mathbf{w}}
$$

where $Z_{\text {act }}^{i}$ is the active antenna impedance of the $i$ th element in the array, $Z_{i n}^{L N A}$ is the low-noise amplifier input impedance, $\mathbf{Z}^{\text {ant }}$ is the $N \times N$ impedance parameter matrix of the antenna, I is the $N \times N$ identity matrix, $\mathbf{i}_{\bullet \mathbf{i}}$ is the $i$ th column-vector of the identity matrix (i.e., an all-zero vector except for a 1 in position $i$ ), and $\mathbf{w}$ is the beam-forming vector.

The measured active antenna impedances of the 32 elements of the array tile, assuming ideal $150 \Omega$ loading conditions and broadside radiation, were calculated using Equation (5). The corresponding reflection coefficients with respect to $Z_{0}=150 \Omega$ are plotted in Figure 14a. The reflection coefficient of the center element was lower than $-8 \mathrm{~dB}$ in the band of interest. For the case of the other elements, this parameter increased up to $-2.5 \mathrm{~dB}$ in the worst case. This degradation was expected, due to the poorer performance provided by the edge elements. However, the active antenna impedance seen from the input of the amplifiers needs to consider the effect of the own amplifiers. The actual active reflection coefficients (also normalized for $Z_{0}=150 \Omega$ ) of the antenna elements, considering that the array was loaded with the implemented differential low-noise amplifiers, are thus shown in Figure 14b. This second case corresponded to the precise source-impedance condition with which the low-noise amplifiers were loaded, and with which their noise performance was determined. As it could be observed from the graph, the poor input reflection coefficient given by the amplifier in the lower part of the band (see Figure 9) caused a strong mismatching in some of the antenna elements in this frequency range, Equation (5). This effect was even more pronounced at low frequencies, due to the higher mutual-coupling levels. This can be understood with the help of Figure 14, which shows the modeling of the receiving active array in terms of the active antenna impedance of each antenna element. The signal transfer between the antennas was modeled by means of the $S$-parameter matrix ( $S^{\text {ant }}$ ) of the radiating structure with respect to the port impedance, $Z_{0}$.

From Figure 14, it could be inferred that when the differential low-noise amplifier (DLNA) reflection coefficient is poor, there may be a larger amount of power that can be coupled into different antennas in the array. According to Figure 9, the poorest differential low-noise amplifier $s_{11}$ parameter occurred for frequencies lower than $400 \mathrm{MHz}$. Actually, some of the active reflection coefficients had values higher than $0 \mathrm{~dB}$ at these frequencies, which provided a source-impedance condition with unpredictable consequences in the low-noise amplifiers. In fact, the stability was not guaranteed, since the unconditionally stable amplifier ensured stability for source impedances that were inside the Smith chart, not outside, as corresponded to a reflection coefficient higher than one in magnitude. The combination of both effects - antenna mismatching and potential instabilities - seemed a convincing explanation for the extremely high noise level present at low frequencies in the measurement of the implemented active array tile (Figure 13). 


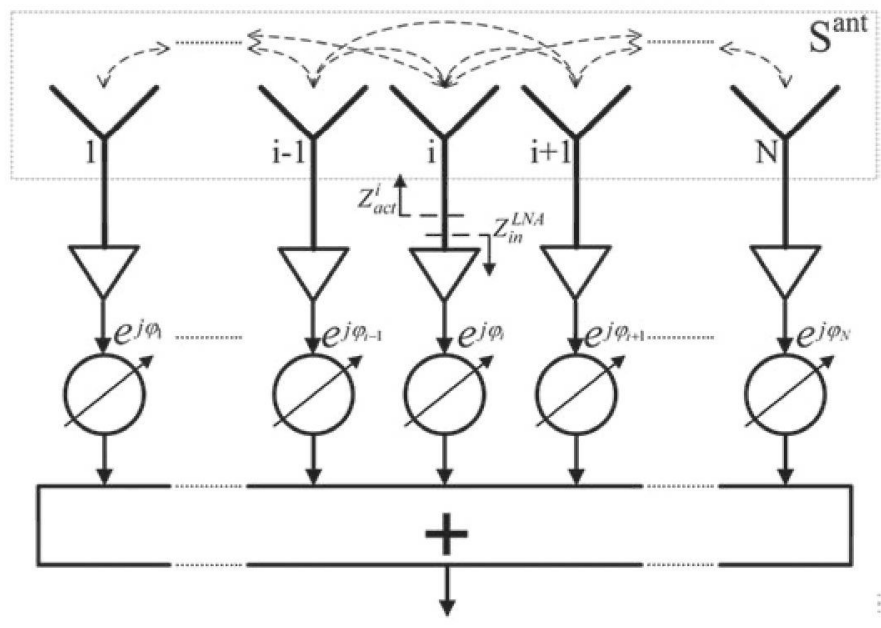

Figure 14. The basic scheme of an $N$-element active-array receiver.

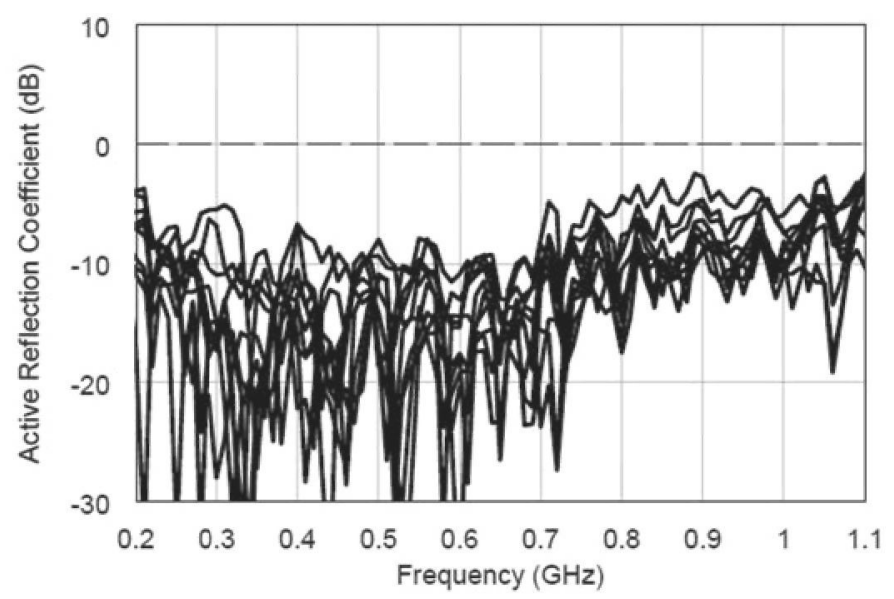

(a)

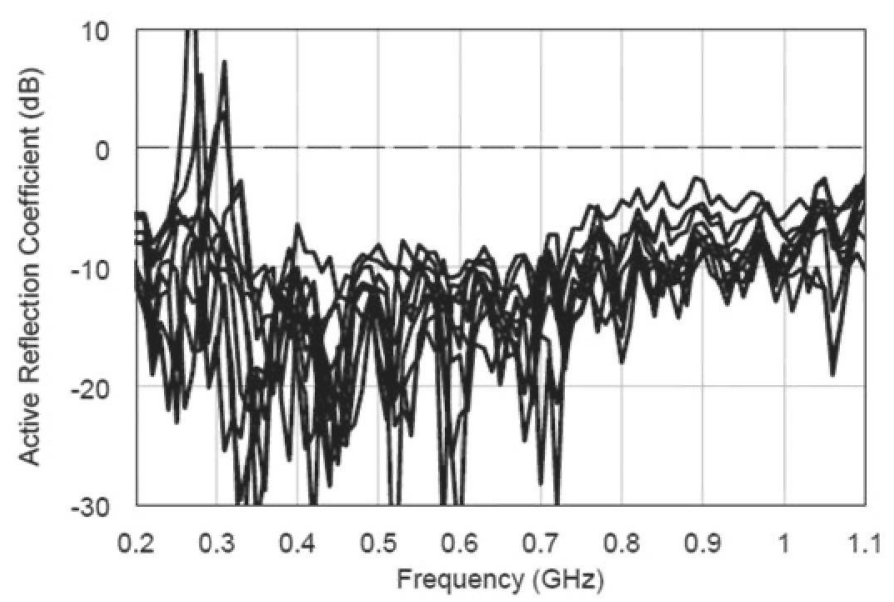

(b)

Figure 15. The measured active reflection coefficients of the 32 elements of the FIDA3 array tile, assuming (a) ideal $150 \Omega$ loading conditions, and (b) loading with the implemented differential low-noise amplifiers.
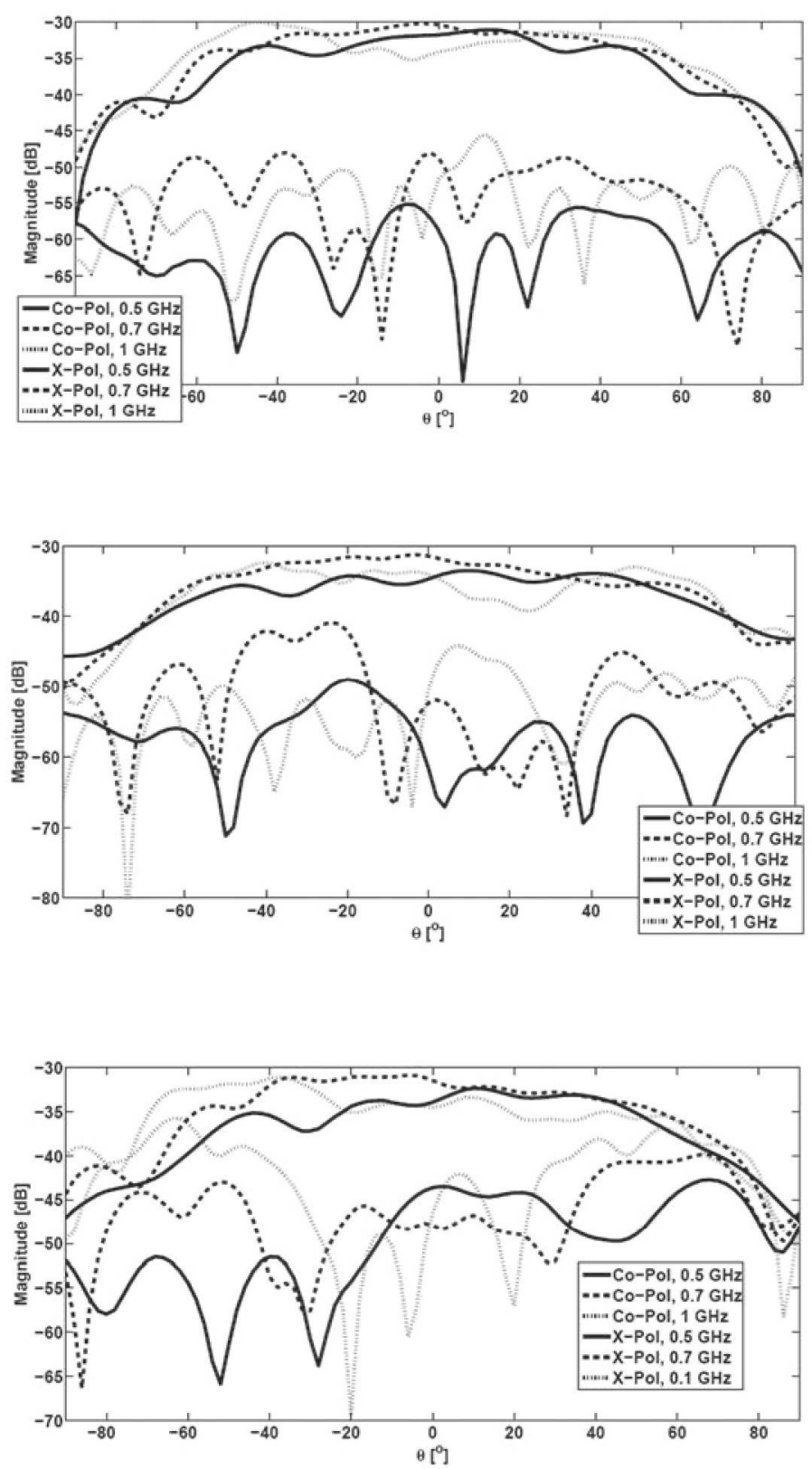

Figure 16. The measured radiation patterns of the embedded element: (top) E-plane pattern, (middle) H-plane pattern, (bottom) diagonal plane. 
Although the previous effects seem critical, they are associated with the small dimensions of the implemented prototype, and can be mitigated with a larger implementation of the array, as is the case for the final SKA stations. The case of an infinite antenna array is equivalent to the case of a singleantenna receiver, since it can be analyzed as a single antenna element with periodic boundary conditions. In both cases, the antenna impedance is non-sensitive to low-noise amplifier variations. In the case of a large antenna array implementation, the active antenna impedances of the elements is expected to converge to a unique value, which corresponds to the value obtainable from an infinite-array simulation (for a given scan angle). As explained before, this last value is independent of the loading conditions (low-noise amplifiers), and effects such as the effect shown in Figure 15b are not going to be present.

Finally, the pattern of the embedded element was included in the $\mathrm{H}$ plane, E plane, and the diagonal plane. It could be seen that the cross-polarized level with respect to the co-polarized level was lower than $15 \mathrm{~dB}$ for scanning angles between $-45^{\circ}$ to $45^{\circ}$.

\section{Conclusion}

The design and characterization process for a differential active-antenna-array demonstrator for radio-astronomy applications has been detailed in this paper. Due to the balanced nature of the wideband antenna elements required for this application, the advantages of using a fully differential receiver are evident. However, some special considerations had to be taken into account with the proposed design. This was because of the anomalies related to the presence of even-mode currents in the balanced antennas, and the ad-hoc measurement methods required to characterize the differential low-noise amplifiers. All of the previous limitations were successfully overcome in this paper, achieving good results with both separated-antenna and low-noise-amplifier implementations. From the complete system integration, the information that could be extracted from the implemented array tile was limited by the reduced size of the prototype. Only in the higher part of the band, did the array began to be large enough to obtain noise values comparable to those expected from the isolated low-noise amplifier measurements. A significantly larger array implementation is thus necessary to obtain a more-reliable idea of the expected noise temperature with the final SKA stations. Nevertheless, implementing a prototype with many more elements presents some strong restrictions in terms of cost, assembly, or characterization. For example, current antenna facilities used to characterize this type of receiving array are not prepared for systems much larger than the implemented $1 \mathrm{~m}^{2}$ tile. On the other hand, the difficulties of characterizing a system in the $300 \mathrm{MHz}$ to $1000 \mathrm{MHz}$ band were evident from the measured results, due to the effects of the external interference in this frequency range. In any case, the information extracted from the implementation and characterization of the present antennaarray prototype was very valuable for the technology choice of the final SKA design.

\section{Acknowledgment}

The authors wish to thank the Netherlands Institute for Radio Astronomy (ASTRON) for its support during the measurement process of the array tile. This work was supported by the European Community Framework Programme 6, Square Kilometre Array Design Studies (SKADS), contract No. 011938 and TEC2009-14525-C02-01.

\section{References}

1. P. E. Dewdney, P. J. Hall, R. T. Schilizzi, and T. J. W. Lazio, "The Square Kilometre Array," Proceedings of the IEEE, 97, 8, August 2009, pp. 482-1496.

2. Square Kilometre Array telescope Web page: http://www. skatelescope.org/.

3. A. Faulkner et al., "The Aperture Arrays for the SKA: The SKADS White Paper," SKA Memo 122, April 2010, available online: http://www.skatelescope.org/.

4. A. B. Smolders and G. W. Kant, "Thousand Element Array (THEA)," IEEE International Symposium on Antennas and Propagation, Salt Lake City, USA, July 2000, 1, pp. 162-165.

5. A. van Ardenne, P. N. Wilkinson, P. D. Patel, and J. G. bij de Vaate, "Electronic Multi-Beam Radio Astronomy Concept: Embrace a Demonstrator for the European SKA Program," Experimental Astronomy, 17, June 2004, pp. 65-77.

6. R. Maaskant, M. V. Ivashina, O. Iupikov, E. A. Redkina, S. Kasturi, and D. H. Schaubert, "Analysis of Large MicrostripFed Tapered Slot Antenna Arrays by Combining Electrodynamic and Quasi-Static Field Models," IEEE Transactions on Antennas and Propagation, AP-59, 6, June 2001, pp. 17981807.

7. L. E. Garcia-Muñoz, O. Garcia-Perez, V. Gonzalez-Posadas, J. L. Vazquez-Roy, D. Segovia-Vargas, J. M. Serna-Puente, T. Finn, and J. A. Lopez-Fernandez, "FIDA3: A Novel Active Array Design for the Mid-frequency Range of the Square Kilometre Array," 4th European Conference on Antennas and Propagation, Barcelona, Spain, April 2010, pp. 1-4.

8. M. Arts, R. Maaskant, E. Lera-Acedo, and J. G. bij de Vaate, "Broadband Differentially Fed Tapered Slot Antenna Array for Radio Astronomy Applications," 3rd European Conference on Antennas and Propagation, Berlin, Germany, March 2009, pp. 566-570.

9. D. M. Pozar, "The Active Element Pattern," IEEE Transactions on Antennas and Propagation, AP-42, 8, August 2009, pp. 1176-1178. 
10. R. Maaskant and E. E. M. Woestenburg, "Applying the Active Antenna Impedance to Achieve Noise Match in Receiving Antenna Arrays," IEEE International Symposium on Antennas and Propagation, Honolulu, USA, June 2007, pp. 5889-5892.

11. E. Lera-Acedo, L. E. Garcia-Muñoz, V. Gonzalez-Posadas, J. L. Vazquez-Roy, R. Maaskant, and D. Segovia-Vargas, "Study and Design of a Differentially Fed Tapered Slot Antenna Array," IEEE Transactions on Antennas and Propagation, AP58, 1, January 2010 , pp. 68-78.

12. O. Garcia-Perez, D. Segovia-Vargas, L. E. Garcia-Muñoz, J. L. Jimenez-Martin, and V. Gonzalez-Posadas, "Broadband Differential Low Noise Amplifier for Active Differential Arrays," IEEE Transactions on Microwave Theory and Techniques, MTT-59, 1, January 2011, pp. 108-115.

13. W. R. Eisenstadt, B. Stengel, and B. M. Thomson, Microwave Differential Circuit Design Using Mixed-Mode S-Parameters, Norwood, MA, Artech House, 2006.

14. J. Morawietz, R. H. Witvers, J. G. Bij de Vaate, and E. E. M. Woestenburg, "Noise Characterization of Ultra Low Noise Differential Amplifiers for Next Generation Radiotelescopes," European Microwave Conference, Munich, Germany, October 2007, pp. 1570-1573.

15. Agilent Technologies, "Noise Figure Measurement Accuracy - Y-Factor Method," Application Note 57-2, October 2004.

16. R. Maaskant and B. Enthoven, "Design of a Hot-cold Test Facility," ASTRON Technical Report SKA-ASTRON-RP-397, 2009.

17. B. Woestenburg, "Definition of Array Receiver Gain and Noise Temperature," SKA Memo 98, February 2008, available online: http://www.skatelescope.org/.

18. C. Craeye, B. Parvais, and X. Dardenne, "MoM Simulation of Signal-to-Noise Patterns in Infinite and Finite Receiving Antenna Arrays," IEEE Transactions on Antennas and Propagation, AP-52, 12, December 2004, pp. 3245-3256.

19. P. Hannan and M. Balfour, "Simulation of a Phased-array Antenna in Waveguide," IEEE Transactions on Antennas and Propagation, AP-13, 3, May 1965, pp. 342-354. 